\title{
IL-37b alleviates endothelial cell apoptosis and inflammation in Kawasaki disease through IL-1R8 pathway
}

Chang Jia', Yingzhi Zhuge ${ }^{2}$, Shuchi Zhang ${ }^{2}$, Chao Ni², Linlin Wang ${ }^{2}$, Rongzhou Wu², Chao Niu', Zhengwang Wen², Xing Rong', Huixian Qiu ${ }^{2}$ and Maoping Chu (id,2

\begin{abstract}
Kawasaki disease (KD) is an acute vasculitis of pediatric populations that may develop coronary artery aneurysms if untreated. It has been regarded as the principal cause of acquired heart disease in children of the developed countries. Interleukin (IL)-37, as one of the IL-1 family members, is a natural suppressor of inflammation that is caused by activation of innate and adaptive immunity. However, detailed roles of IL-37 in KD are largely unclear. Sera from patients with KD displayed that IL-37 level was significantly decreased compared with healthy controls (HCs). QRT-PCR and western blot analyses showed that the expression level of IL-37 variant, IL-37b, was remarkably downregulated in human umbilical vein endothelial cells (HUVECs) exposed to KD sera-treated THP1 cells. Therefore, we researched the role of IL-37b in the context of KD and hypothesized that IL-37b may have a powerful protective effect in KD patients. We first observed and substantiated the protective role of IL-37b in a mouse model of KD induced by Candida albicans cell wall extracts (CAWS). In vitro experiments demonstrated that IL-37b alleviated endothelial cell apoptosis and inflammation via IL-1R8 receptor by inhibiting ERK and NFKB activation, which were also recapitulated in the KD mouse model. Together, our findings suggest that IL-37b play an effective protective role in coronary endothelial damage in KD, providing new evidence that IL-37b is a potential candidate drug to treat KD.
\end{abstract}

\section{Introduction}

Kawasaki disease (KD) is an illness that can cause systemic immune vasculitis, which mainly has an effect on young children. The most severe complication of KD is coronary artery injury, including coronary artery dilation, coronary artery aneurysm (CAA) and stenosis. Clinically, the state-of-the-art therapeutic strategy is intravenous immunoglobulin (IVIG) plus aspirin. In the absence of early intervention, about $25 \%$ of KD patients

\footnotetext{
Correspondence: Huixian Qiu (qiuhx1053@126.com) or

Maoping Chu (chmping@hotmail.com)

${ }^{1}$ Pediatric Research Institute, The Second Affiliated Hospital and Yuying

Children's Hospital of Wenzhou Medical University, 325027 Wenzhou, China

${ }^{2}$ Children's Heart Center, Institute of Cardiovascular Development and

Translational Medicine, The Second Affiliated Hospital and Yuying Children's

Hospital of Wenzhou Medical University, 325027 Wenzhou, China

These authors contributed equally: Chang Jia, Yingzhi Zhuge

Edited by B. Zhivotovsky
}

would develop CAA. Even with timely treatment, there is still unacceptable high risk of giving rise to CAA. Therefore, it is urgent to explore novel and potential therapeutic strategy to clinically treat $\mathrm{KD}$ and its complications.

Interleukin-37 (IL-37) is a newly reported member of the IL-1 family and functions as a novel anti-inflammatory cytokine to suppress inflammatory and immune response. Studies report that IL-37 plays an essential role in mediating inflammatory diseases, including arthritis ${ }^{1}$, liver inflammation ${ }^{2}$, and cardiovascular disease inflammation ${ }^{3}$. In addition, increasing evidence demonstrate that exogenous IL-37 can ameliorate cardiovascular injury, including vascular calcification, atherosclerosis ${ }^{4}$, aortic valve lesion $^{5}$, myocardial infraction ${ }^{6}$, and myocardial ischemia/reperfusion injury ${ }^{7}$. Currently, five splice variants of human IL-37 (IL-37a-e) have been identified,

\section{(c) The Author(s) 2021, corrected publication 2021}

(c) (i) Open Access This article is licensed under a Creative Commons Attribution 4.0 International License, which permits use, sharing, adaptation, distribution and reproduction cc) in any medium or format, as long as you give appropriate credit to the original author(s) and the source, provide a link to the Creative Commons license, and indicate if changes were made. The images or other third party material in this article are included in the article's Creative Commons license, unless indicated otherwise in a credit line to the material. If material is not included in the article's Creative Commons license and your intended use is not permitted by statutory regulation or exceeds the permitted use, you will need to obtain permission directly from the copyright holder. To view a copy of this license, visit http://creativecommons.org/licenses/by/4.0/. 
with IL-37b being the largest and most investigated. Extracellular IL-37 and its splice variants IL-37b and IL$37 \mathrm{~d}$ are reported to mitigate inflammation via binding to IL-1R $8^{3,8-12}$. IL-1R8, also termed as single immunoglobulin G (IgG) IL-1-related receptor, has merely a single Ig domain and is able to provide a brake on inflammation ${ }^{13}$. Presently, whether KD vasculitis can be ameliorated by IL-37 and its subtypes is still unidentified.

As is known, endothelial cell (EC) injury and inflammation are the key pathological mechanisms for KD, and alleviating endothelial injury or inflammation can be an effective mean to treat $\mathrm{KD}^{14-16}$. IL-37 exerts antiinflammatory response in many types of cells, including $\mathrm{ECs}^{17}$. Recent studies revealed that IL-37 inhibit inflammatory response in human coronary artery ECs via intracellularly suppressing the Toll-like receptor 2nuclear factor $\kappa B(\mathrm{NF} \kappa \mathrm{B})$-intercellular adhesion molecule 1 (ICAM-1) pathway ${ }^{17}$. In addition to antiinflammatory activity, IL-37 is also related to apoptosis. For instance, $\mathrm{Li}$ et al. reported that IL-37 attenuates lipopolysaccharide (LPS)-induced neonatal acute respiratory distress syndrome in young mice via inhibiting inflammation and cell apoptosis ${ }^{18}$. Wu et al. demonstrated that mice treated with IL-37 show an obvious amelioration of the ischemia/reperfusion injury, as demonstrated by reduced infarct size, decreased cardiac troponin $\mathrm{T}$ level, and improved cardiac function. This protective effect was associated with the ability of IL-37 to suppress production of pro-inflammatory cytokines, chemokines, and neutrophil infiltration, which together contributed to a decrease in cardiomyocyte apoptosis and reactive oxygen species generation ${ }^{7}$. Until now whether IL-37 can inhibit EC apoptosis, especially in KD, remains still unknown.

To investigate the effect of IL-37 on KD, we first examined the level of IL-37 in the serum from KD patients as compared with healthy controls (HCs) and affirmed that its splice variant IL-37b might function in KD. Then we investigated the therapeutic effect of IL-37b on KD through in vivo and in vitro experiments. Ultimately, we substantiated that IL-37b alleviated KD through IL-1R8 pathway by inhibiting the activation of extracellular signal-regulated kinase (ERK) and NFкB. This study may provide a potentially therapeutic target for KD coronary endothelial injury.

\section{Results}

IL-37b expression level was significantly decreased in KD serum-treated human umbilical vein endothelial cells (HUVECs)

To preliminarily confirm that IL-37 might be involved in the pathological process of $\mathrm{KD}$, we first examined the level of IL-37 in the serum from KD patients compared with the HCs. Enzyme-linked immunosorbent assay (ELISA) showed that the serum level of IL-37 was much lower in KD patients (Fig. 1a). Next, the mRNA expression levels of five splice variants of IL37 gene, IL37a-e, were determined in KD serum-treated ECs using quantitative real-time polymerase chain reaction (qRT-PCR) analysis. Results showed that only $I L 37 b$ was significantly

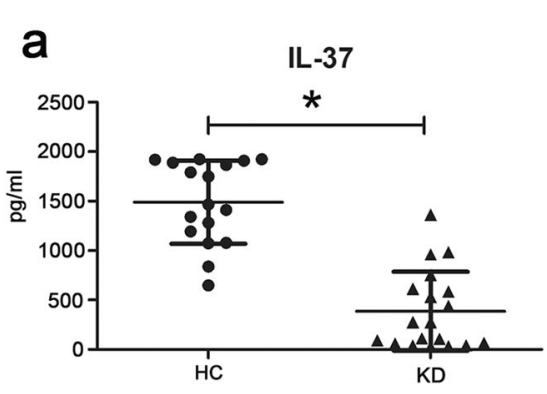

b
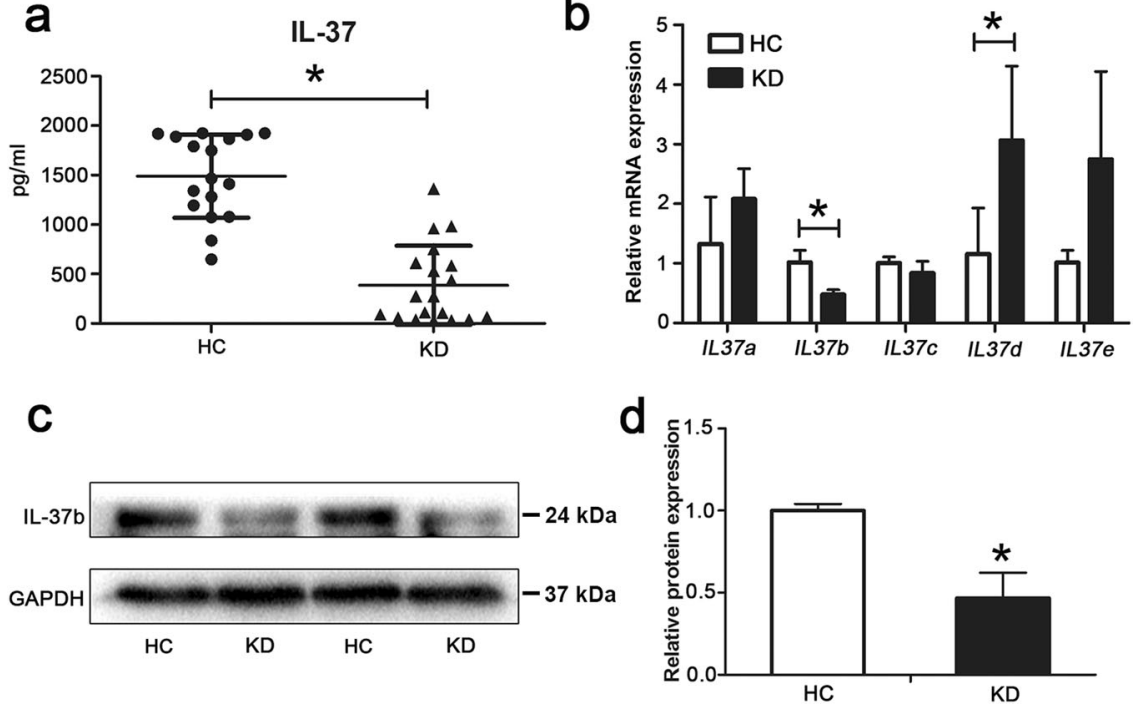

Fig. 1 The expression level of IL-37b was decreased after KD sera treatment. a The levels of IL-37 in sera from health controls (HCs, $n=17)$ and KD patients $(n=18)$ were evaluated by enzyme-linked immunosorbent assay (ELISA). Significance: ${ }^{*} P<0.05$. $\mathbf{b}$ The mRNA expression levels of five IL37 splice variants (IL-37a-e) were evaluated in KD-treated ECs by qRT-PCR analysis. Significance: ${ }^{*} P<0.05$. $\mathbf{c}$, $\mathbf{d}$ Protein expression of IL-37b was assessed in KD-treated ECs by western blot analysis. GAPDH was used as an internal control. Quantitative analysis of IL-37b expression was performed (d). All these experiments were repeated at least three times. Data are shown as mean \pm SD $(n=3)$. Significance: ${ }^{*} P<0.05$. 

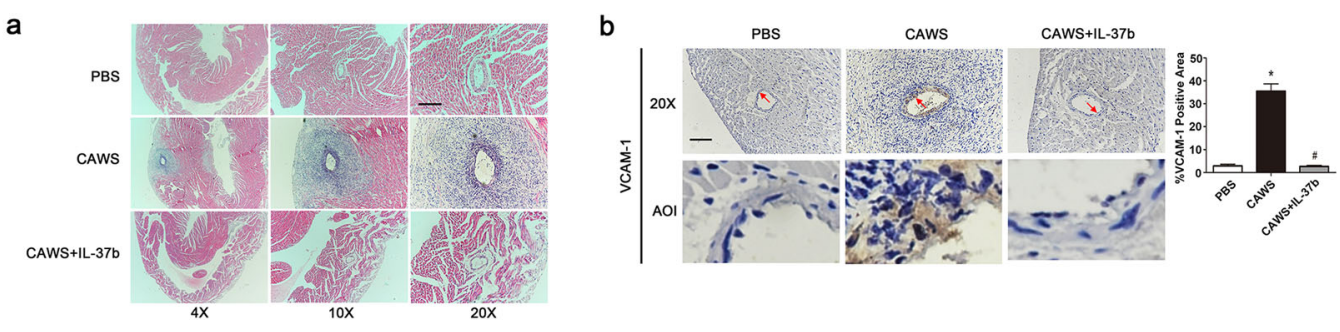

c
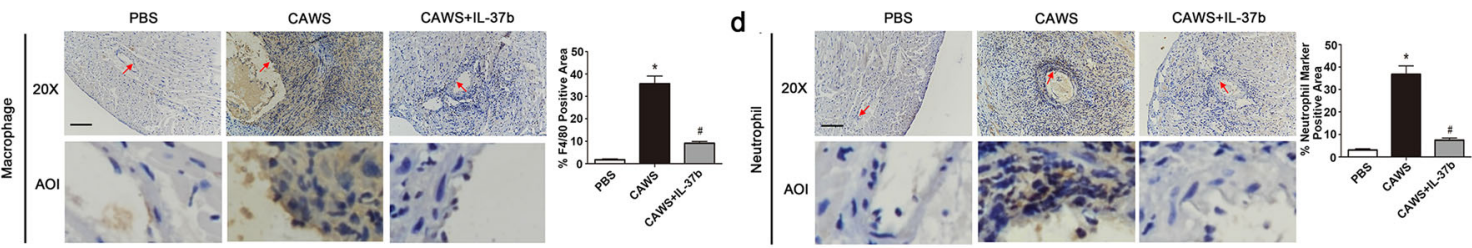

e

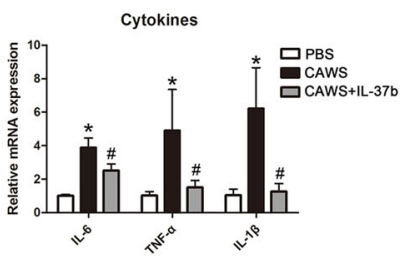

f

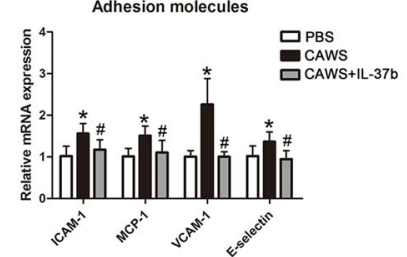

g

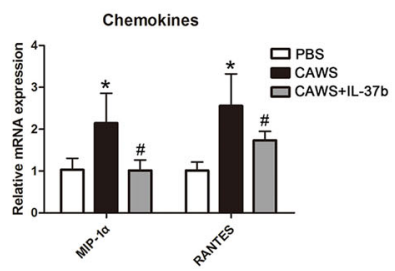

Fig. 2 The coronary arteritis was mitigated after II-37b injection in a KD mouse model. a Representative images from H\&E staining at 28-day post-CAWS injection. Magnification: $\times 200$. Scale bar $=100 \mu \mathrm{m}$. b-d The expression levels of vascular cell adhesion molecule 1 (VCAM-1), macrophage marker F4/80, and neutrophil marker were, respectively, determined using $\mathrm{HC}$ staining in the endothelium of coronary arteries. Enlarged images of area of interesting (AOI) were indicated with a red arrow. Scale bar $=100 \mu \mathrm{m}$. e- $\mathbf{g}$ The expression levels of cytokines, adhesion molecules, and chemokines were detected in the mice heart section. Significance: ${ }^{*} P<0.05$ vs. the PBS group, and ${ }^{\#} P<0.05$ vs. the CAWS group. N $=6$ mice per group, and each experiment was conducted at least three times.

downregulated in KD serum-treated ECs compared with HC serum-treated ECs (Fig. 1b). Western blot analysis further substantiated that the protein expression level of IL-37b was decreased after KD serum treatment (Fig. 1d). Taken together, the expression of IL-37b was downregulated in ECs after KD serum treatment.

\section{Recombinant human IL-37b alleviates coronary artery injury in a KD mouse model}

IL-37b has been reported to have potent antiinflammatory activities in autoimmune diseases ${ }^{19,20}$. However, its function in KD is still unclear. To initially investigate the role of IL-37b, recombinant human IL-37b was intraperitoneally injected into Candida albicans cell wall extract (CAWS)-induced KD mouse model. As expected, treatment with IL-37b significantly attenuated coronary artery inflammation (Fig. 2a). As is known, vascular cell adhesion molecule 1 (VCAM-1) is an essential molecule to mediate the adhesion of many leukocytes to the vascular endothelium, the elevated expression of which is regarded as a marker for EC activation during vascular inflammation ${ }^{21}$. As shown in Fig. $2 \mathrm{~b}$, immunohistochemistry (IHC) analysis demonstrated treatment of mice with CAWS significantly upregulated the expression of VCAM-1 in the intima of coronary artery, coupled with increased infiltration of inflammatory cells involved in macrophages and neutrophils (Fig. 2c, d). In addition, the expression of inflammation-related factors, including cytokines, adhesion molecules, and chemokines, were also upregulated in the KD mouse model (Fig. 2e-g), consistent with previous studies ${ }^{22}$. However, IL-37b injection remarkably decreased VCAM-1 expression levels, inflammatory cell adhesion, and expression of inflammation-related factors, indicating that IL-37b possesses a good anti-inflammatory effect in KD.

\section{Recombinant human IL-37b mitigated EC apoptosis and inflammation}

Previous studies and the above in vivo results demonstrated that vascular ECs were damaged in $\mathrm{KD}^{21,23}$, and IL-37b could alleviate EC injury and the subsequent coronary artery inflammation. Considering ECs are exposed to inflammatory environment in $\mathrm{KD}$, we utilized KD sera-treated THP1 cells and co-cultivated them with HUVECs to create an in vitro coculture experimental system as described by our previous studies ${ }^{16}$. To investigate the protective efficacy of IL-37b, different concentrations of recombinant human IL-37b were added into ECs before co-culturing them with KD sera-treated THP1 cells (referred to as "KD-treated ECs" from now 
a

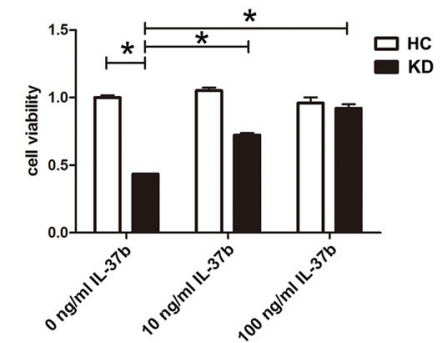

e

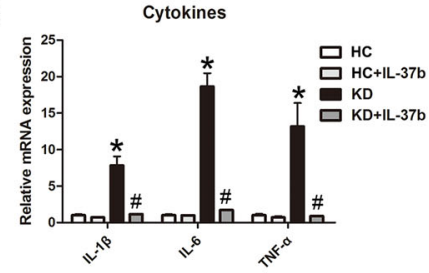

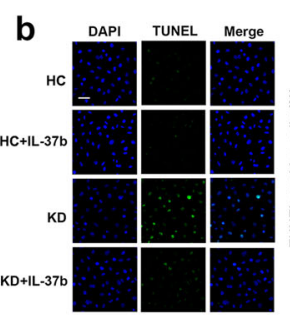

f

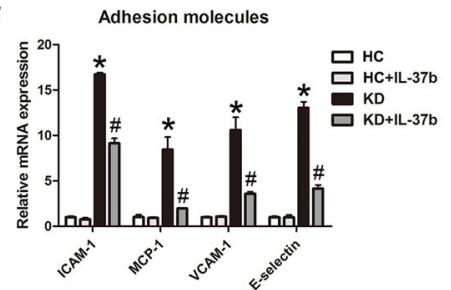

$\begin{array}{lll}\mathrm{HC} & \stackrel{\mathrm{HC}+}{\mathrm{LL}-37 \mathrm{~b}} & \mathrm{KD}+ \\ \mathrm{KD} & \mathrm{IL}-37 \mathrm{~b}\end{array}$

C

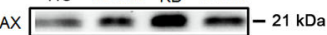

$\mathrm{BCl}-2=-26 \mathrm{kDa}$

$\mathrm{GAPDH}=-37 \mathrm{kDa}$

d

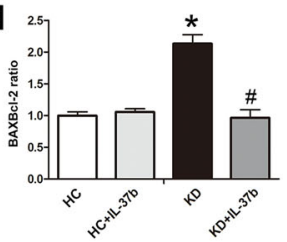

g

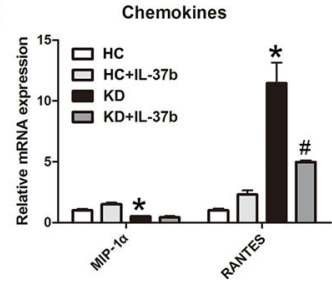

Fig. 3 IL-37b alleviated KD-treated endothelial cell apoptosis and inflammation. a CCK8 assay was used to evaluate cell viability ( $n=6$ ) Significance: ${ }^{*} P<0.05$. b DNA fragmentation was analyzed using TUNEL staining $(n=3)$. c Protein expression of BAX and Bcl-2 was determined by western blot analysis. $\mathbf{d}$ Quantitative analysis of BAX/BCl-2 ratio was conducted. Data are presented as mean $\pm \mathrm{SD}(n=3)$. e, $\mathbf{f}$ The expression levels of cytokines, adhesion molecules, and chemokines were examined in KD-treated ECs after IL-37b treatment $(n=3)$. ${ }^{*} P<0.05$ vs. the HC group, and ${ }^{\#} P<$ 0.05 vs. the KD group. At least three experiments were performed for each assay.

on). Cell Counting Kit-8 (CCK8) analysis exhibited that IL-37b markedly rescued cell viability, and $100 \mathrm{ng} / \mathrm{ml} \mathrm{IL}-$ 37b had the best rescue effect (Fig. 3a). Therefore, $100 \mathrm{ng} /$ $\mathrm{ml}$ IL-37b was chosen to treat cells in the follow-up experiments. Studies reported that IL-37 can improve the survival rate of podocytes through suppressing high glucose-induced apoptosis ${ }^{24}$. Therefore, we examined the effect of IL-37b on EC apoptosis. Data displayed that IL37b significantly inhibited KD sera-induced EC apoptosis, as evidenced by declined percentage of terminal deoxynucleotidyl transferase-mediated dUTP-fluorescein nick end labeling (TUNEL)-positive cells and downregulated $\mathrm{BAX} / \mathrm{Bcl}-2$ ratio compared with the $\mathrm{KD}$-treated EC group (Fig. 3b-d). In addition, we found that IL-37b treatment significantly decreased the expression levels of inflammation-related factors in KD-treated ECs, including IL-1 $\beta$, IL-6, tumor necrosis factor (TNF)- $\alpha$, ICAM-1, monocyte chemotactic protein-1, VCAM-1, E-selectin, and RANTES (regulated and normal $\mathrm{T}$ cell expressed and secreted; Fig. $3 \mathrm{e}-\mathrm{g}$ ). The above data indicate that IL-37b addition can inhibit KD-treated EC apoptosis and inflammation.

\section{IL-37b-mediated inhibition of EC apoptosis and inflammation was associated with IL-1R8}

Previous studies reported that extracellular forms of IL-37 function via the IL-1 family decoy receptor IL$1 \mathrm{R} 8^{25,26}$. To further investigate the mechanism of IL-
$37 \mathrm{~b}$ in $\mathrm{KD}$, the mRNA expression level of IL-1R8 was determined. Results showed that treatment with KD serum significantly upregulated IL-1R8 expression, and addition of IL-37b further elevated its expression (Fig. 4a). As reported, IL-1R8 will transfer onto the cell surface to bind with IL-37 upon stimulation ${ }^{25}$. Herein the surface expression and intracellular expression of IL-1R8 were measured. As shown in Fig. 4b, c, intracellular IL-1R8 remained unchanged in these groups. However, the expression of surface IL-1R8 was notably increased in KDtreated ECs, and IL-37b supplementation further upregulated surface IL-1R8 expression, which was consistent with the mRNA results, indicating that IL-37b might function via IL-1R8. To confirm this, HUVECs were transfected with empty plasmid (negative control) or IL-1R8 siRNA. Data showed that IL-1R8 siRNA remarkably silenced the expression of IL-1R8 (Fig. 4d). Moreover, the induced upregulation of IL-1R8 in KD-treated ECs was notably suppressed by IL-1R8 siRNA (Fig. 4e). The expression change of IL-1R8 was also substantiated at the protein level (Fig. 4f, g). Next, the effects of IL-1R8 silencing on EC apoptosis and inflammation were analyzed. As anticipated, the inhibition effect of IL-37b on apoptosis was significantly reversed upon IL-1R8 silencing, as demonstrated by increased percentage of TUNEL-positive cells and elevated $\mathrm{BAX} / \mathrm{Bcl}-2$ ratio (Fig. $4 \mathrm{~h}-\mathrm{j}$ ). In addition, the decreased expression of TNF- $\alpha$, IL- $1 \beta$, and IL- 6 mediated by IL-37b were also distinctly abolished by silencing of IL- 


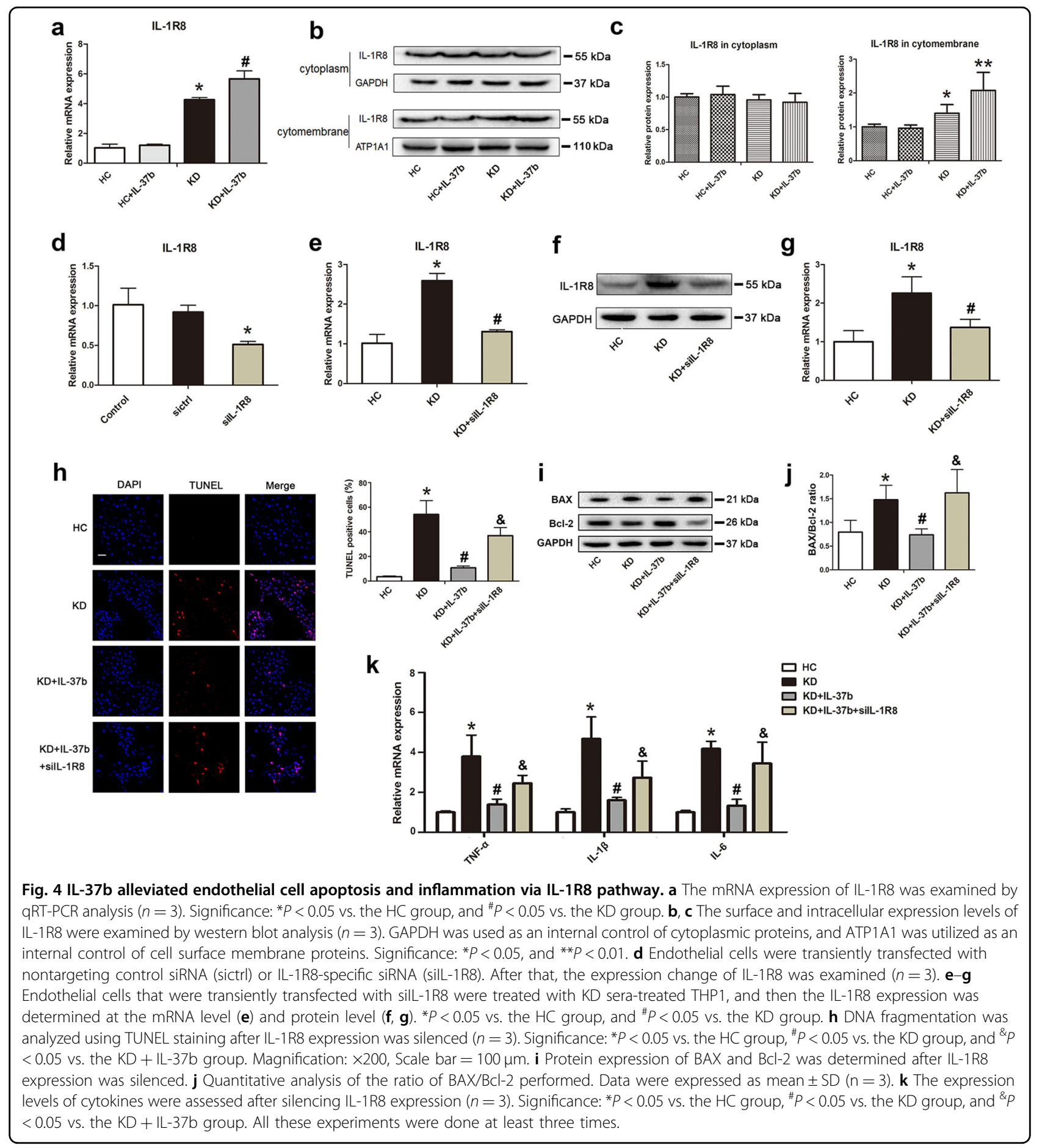

1R8 (Fig. 4k). Taken together, these results provided strong evidence that IL-37b inhibited KD-treated EC apoptosis and inflammation via IL-1R8 pathway.

\section{Recombinant IL-37b inhibited ERK and NFKB activation in KD-treated ECs}

The intracellular signal transduction network showed that IL-37 can mediate a decrease in the components of the NFkB pathway, including $\mathrm{p} 65^{27}$. Studies reported that IL-37b can inhibit mitogen-activated protein kinase (MAPK), including p38, c-Jun N-terminal kinase (JNK), and ERK, and NFKB p65 activation in response to IL-1 $\beta$ related to IL-1R8 ${ }^{9}$. As is known, MAPK and NFkB signaling pathways are able to modulate cell apoptosis and inflammation ${ }^{28}$. Therefore, we first examined the activation of ERK, JNK, p38, and NFkB p65. As shown in Fig. 


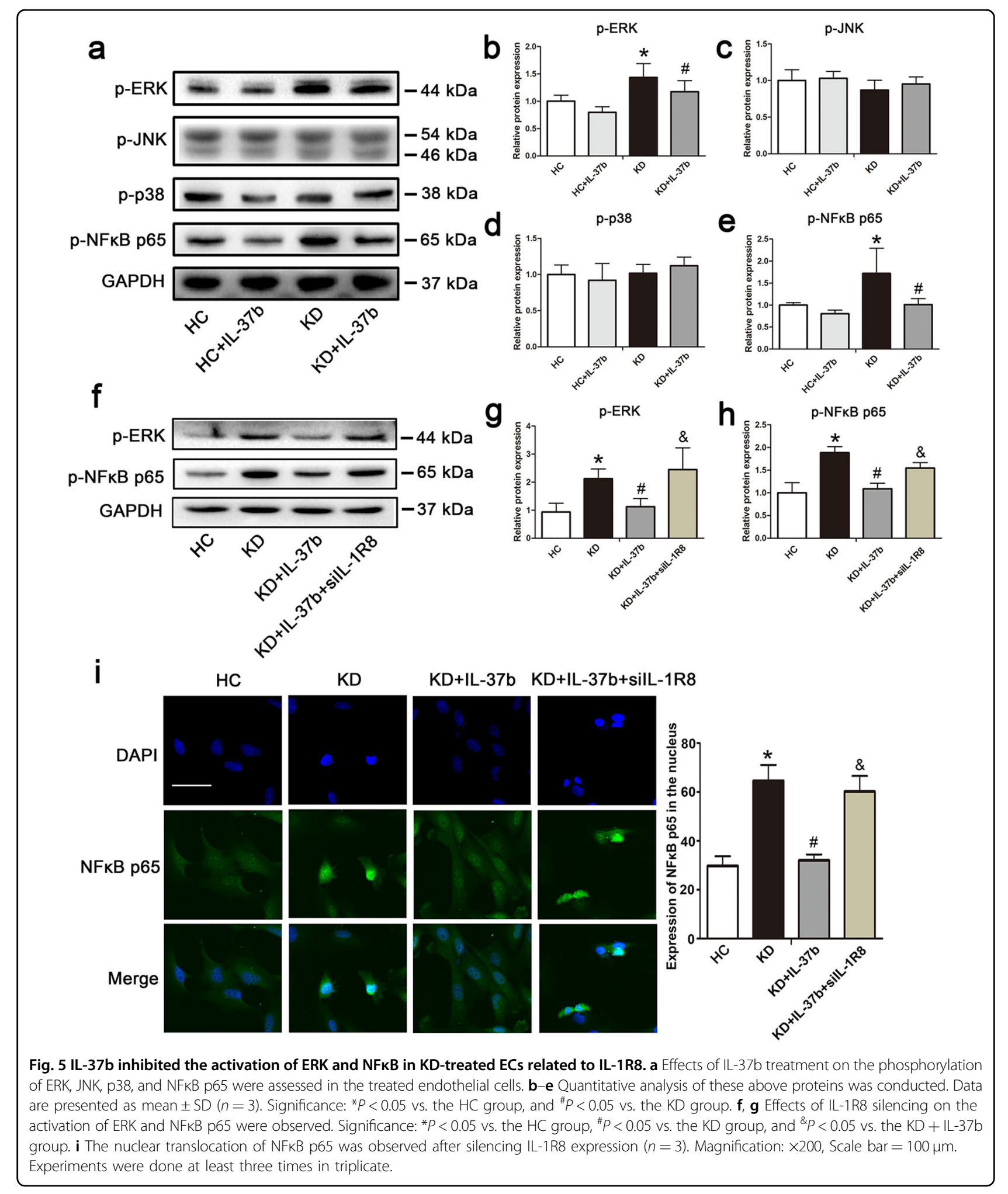

5a-e, ERK and NFKB p65 were significantly phosphorylated and activated after KD sera treatment. However, addition of IL-37b remarkably decreased the phosphorylation levels of ERK and NFKB p65, indicating that IL-37b could suppress the activation of these two proteins. To further substantiate that IL-37b modulated 


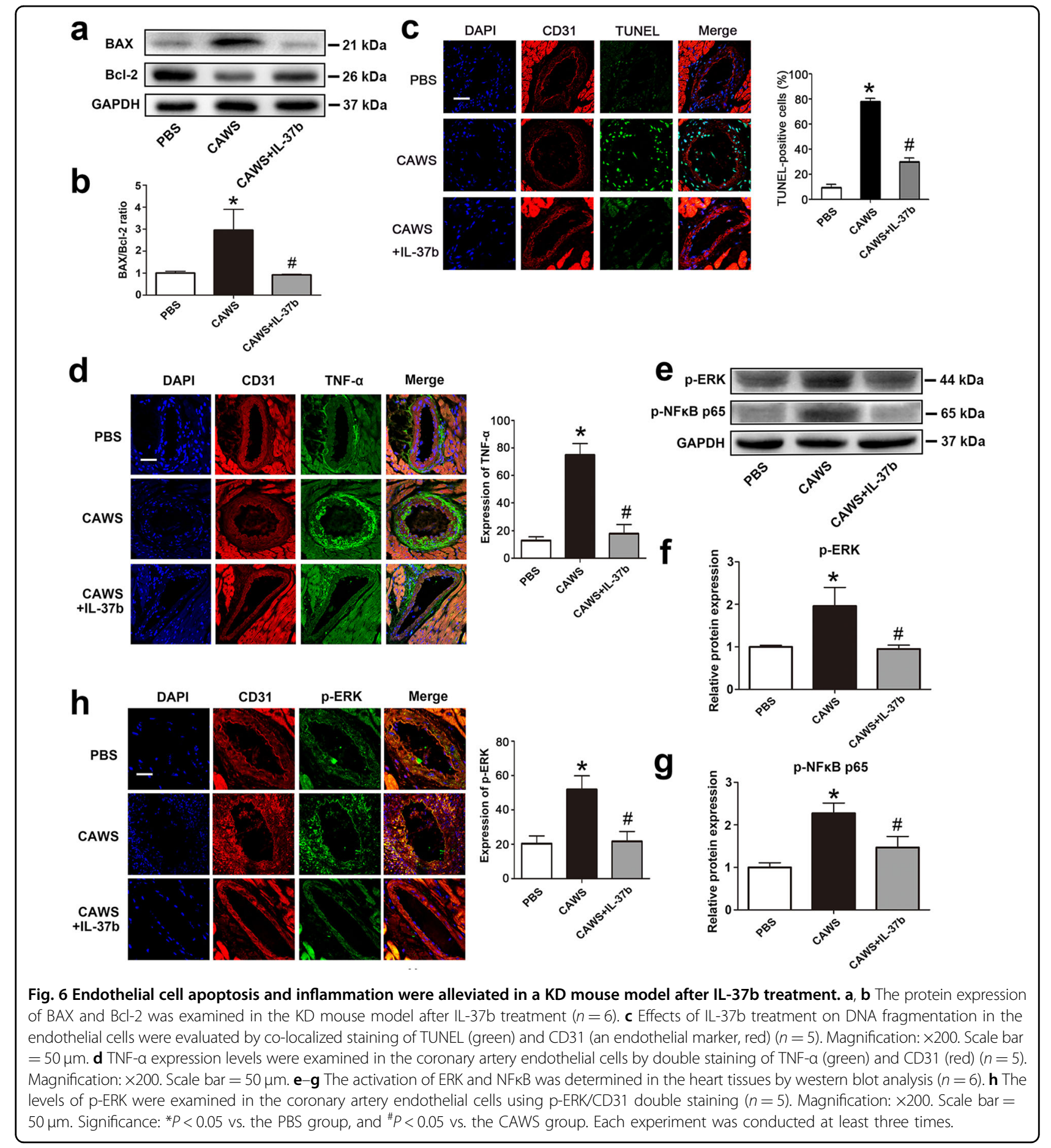

the activation of ERK and NFKB p65 via IL-1R8, IL-1R8 was silenced. As expected, a decrease in IL-1R8 expression significantly reversed the phosphorylation levels of ERK and NFKB p65 (Fig. 5f-h). Immunofluorescence staining showed that $\mathrm{NF \kappa B}$ p 65 translocation into the nucleus was attenuated upon IL-37b treatment.
However, the translocation of NFkB p65 into the nucleus was enhanced after silencing of IL-1R8 compared with the IL-37b treatment group (Fig. 5i), further disclosing that IL-37b suppressed the activation of NFKB. Together, these data indicate that IL-37b treatment can affect the activation of ERK and NFkB. 


\section{Recombinant IL-37b attenuated EC apoptosis and inflammation in a KD mouse model}

To further explore the in vivo protective mechanism of IL-37b, the expression levels of apoptosis-associated proteins $\mathrm{BAX}$ and $\mathrm{Bcl}-2$ were examined in the heart tissue from the KD mouse model. As shown in Fig. 6a, b, the ratio of $\mathrm{BAX} / \mathrm{Bcl}-2$ was significantly decreased upon recombinant IL-37b treatment. To further substantiate the effect on apoptosis occurring in coronary artery endothelium, CD31/TUNEL double staining was conducted. As shown in Fig. 6c, the percentage of TUNELpositive cells in coronary artery endothelium of the KD mouse model was markedly reduced by IL-37b. In addition, the expression of TNF- $\alpha$ and IL-18 in the endothelium of coronary artery was also remarkably downregulated by IL-37b, as evidenced by decreased immunofluorescence intensity after CD31/TNF- $\alpha$ or CD31/IL-18 double staining (Fig. 6d and Fig. S2e). Moreover, the elevated plasma concentrations of TNF- $\alpha$, IL-1 $\beta$, IL-6, and IL-18 in the KD mouse model were also declined by treatment with IL-37b (Fig. S2a-d). These results indicated that IL-37b treatment significantly alleviated $\mathrm{EC}$ apoptosis and inflammation in the KD mouse model. To further demonstrate the mechanism of IL-37b inhibiting apoptosis and inflammation, the activation of ERK and NFKB p65 was analyzed. As shown in Fig. 6e-g, IL-37b treatment markedly decreased their phosphorylation level, and the immunofluorescence intensity of pERK was notably attenuated (Fig. 6h), indicating that IL$37 \mathrm{~b}$ might mitigate endothelium damage via decreasing ERK and NFKB activation.

\section{Recombinant IL-37b mitigated coronary endothelium damage via IL-1R8 pathway in the KD mouse model}

To in vivo demonstrate whether IL-37b contributed to anti-apoptosis and anti-inflammation functions through IL$1 \mathrm{R} 8$ receptor in KD mouse model, the expression level of IL-1R8 was first determined. Consistent with in vitro results, the mRNA level of IL-1R8 was significantly upregulated in the CAWS group, and addition of IL-37b further increased its expression (Fig. 7a). To assess the role of IL$1 \mathrm{R} 8$ in vivo, an adeno-associated virus 9-mediated RNA interference targeting IL-1R8 (AAV9-IL-1R8 siRNA) was administrated into the KD mouse model in the presence of IL-37b. As shown in Fig. 7b, the inhibition effect of IL-37b on coronary inflammation was remarkably abrogated by IL1R8 gene silencing. Furthermore, silencing of IL-1R8 also upregulated the expression of VCAM-1 and increased infiltration of macrophages and neutrophils into the endothelium compared with the IL-37b addition group (Fig. $7 \mathrm{c}-\mathrm{e})$. To further elucidate the effect of IL-1R8 on EC apoptosis and inflammation in vivo, apoptosis-related parameters and the expression of inflammatory cytokines were determined. Compared with the IL-37b treatment group, the percentage of TUNEL-positive cells in the endothelium and $\mathrm{BAX} / \mathrm{Bcl}-2$ ratio were notably increased upon silencing of IL-1R8 (Fig. $7 \mathrm{f}-\mathrm{h}$ ). Moreover, the downregulation of IL- 6 , TNF- $\alpha$, and IL- $1 \beta$ mediated by IL$37 \mathrm{~b}$ were also affected by IL-1R8 gene silencing (Fig. 7i). In addition, the phosphorylation levels of ERK and NFkB p65 were increased (Fig. 7j-l).

\section{Discussion}

In the current study, we revealed that IL-37b plays a critical protective role against KD and that the protective effect is realized via IL-1R8 pathway, inhibiting ERK and NFKB activation, which results in suppression of EC apoptosis and inflammation (Fig. 8). This previously unappreciated anti-inflammatory cytokine provides new clues to develop potential diagnosis, evaluation, and treatment strategies against KD.

$\mathrm{KD}$ is a kind of acute systemic vasculitis that involves severe inflammatory environment, which is caused by a variety of cells, including ECs. Therefore, targeting endothelial inflammation in KD could be a potential therapeutic strategy. IL-37 has emerged as an effective inhibitor to attenuate inflammation in models of osteoarthritis $^{9}$, colitis ${ }^{29}$, and periodontal inflammation ${ }^{30}$. However, the level of IL-37 and whether it can have an effect on KD are still unidentified.

In this study, we first demonstrated that the level of IL37 in sera from KD patients was significantly decreased compared with HCs. As is known, IL-37 has five spiced variants (IL-37a-e) ${ }^{31}$. Our qRT-PCR analysis showed that the expression of $I L 37 b$ was significantly downregulated in KD serum-treated ECs, which was also substantiated by western blot analysis. However, the mRNA expression level of IL37d was elevated compared with HC serumtreated ECs. Studies reported that IL-37d is a functional cytokine that can negatively modulate the expression of pro-inflammatory cytokines and possesses antiinflammatory roles ${ }^{12,32}$. Upregulation of IL-37d expression in KD serum-treated ECs might be a compensatory response since its elevation did not rescue EC inflammation mediated by KD serum. IL-37b was widely reported to have an anti-inflammatory effect ${ }^{31}$. Our in vivo study also demonstrated that the administration of recombinant human IL-37b significantly inhibited coronary artery inflammation and VCAM-1 expression in the KD mouse model, indicating that IL-37b inhibited EC activation and played a protective role in the pathophysiology of KD. As is known, the innate and adaptive immunity is activated during the acute phase of $K D$, resulting in an increase in cytokine production by immune effector cells. The secreted cytokine damage vascular ECs and induce the expression of leukocyte adhesion molecules on vascular endothelium, finally leading to infiltration of inflammation cells and coronary 


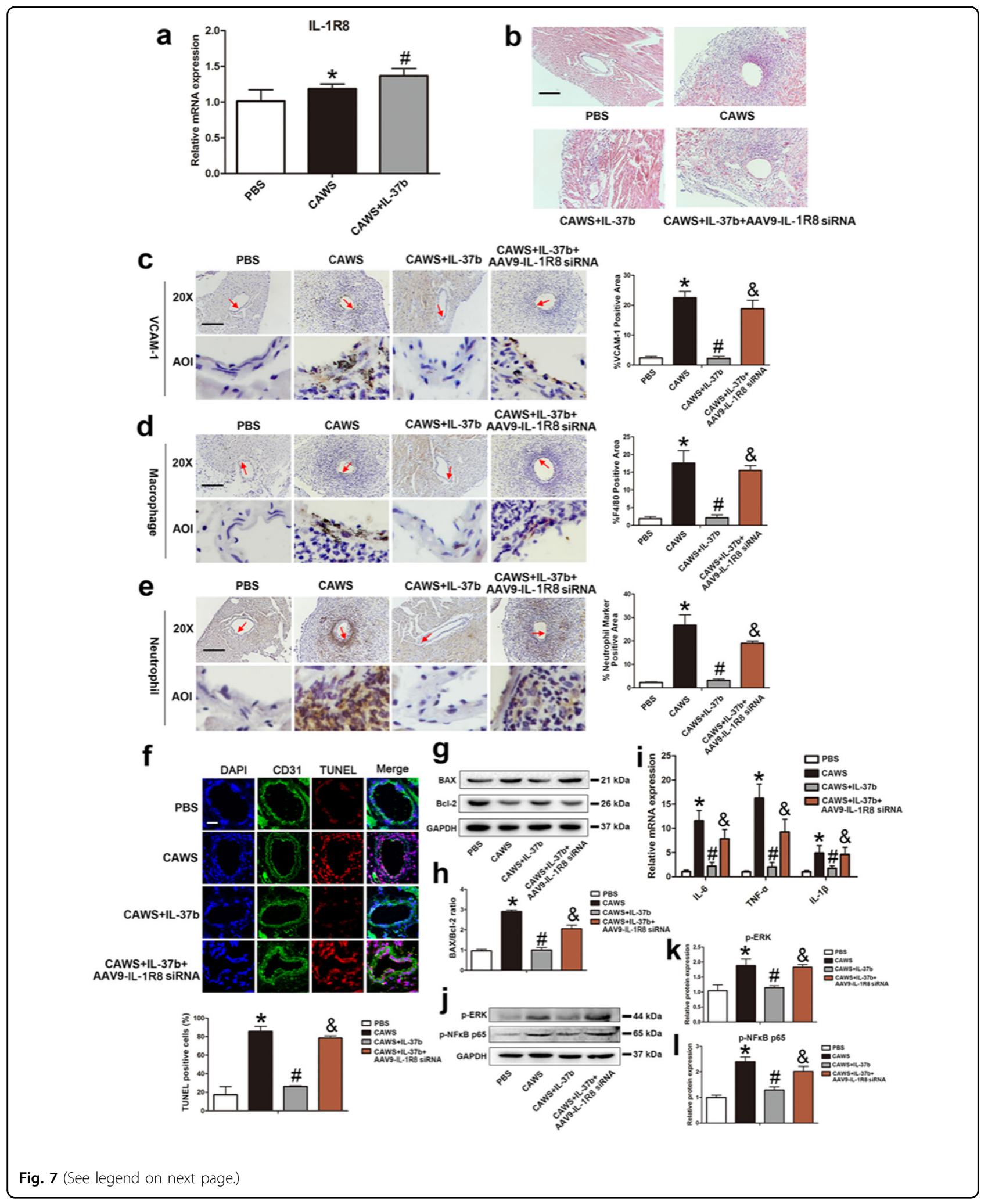


(see figure on previous page)

Fig. 7 Mitigation of IL-37b-mediated coronary artery endothelial cell apoptosis and inflammation was realized via IL-1R8 pathway. a The expression of IL-1R8 were detected in the KD mouse model after IL-37b treatment $(n=6)$. $\mathbf{b}$ Effects of silencing of IL-1R8 on coronary arteritis were observed by H\&E staining. Magnification: $\times 200$. Scale bar $=100 \mu \mathrm{m}$. c-e The endothelial expression of VCAM-1, macrophage marker F4/80, and neutrophil marker was examined after IL-1R8 gene silencing using IHC staining. AOI were indicated with a red arrow. Scale bar $=100 \mu \mathrm{m}$. Right: The histograms respectively exhibited the percentage of VCAM-1, F4/80, and neutrophil marker-positive areas. Significance: ${ }^{*} P<0.05$ vs. the PBS group, ${ }^{\#} P$ $<0.05$ vs. the CAWS group, and ${ }^{\&} P<0.05$ vs. the CAWS + IL-37b group. $f$ DNA fragmentation was examined using TUNEL staining. Scale bar $=$ $100 \mu \mathrm{m}$. Below: Percentage of TUNEL-positive cells was shown in the histogram. Significance: ${ }^{*} P<0.05$ vs. the PBS group, ${ }^{\#} P<0.05$ vs. the CAWS group, and ${ }^{\&} P<0.05$ vs. the CAWS + IL-37b group. $\mathbf{g}, \mathbf{h}$ BAX/BCl-2 ratio was analyzed by western blot analysis. $\mathbf{i}$ The mRNA expression levels of IL-6, TNF- $a$, and IL-1 $\beta$ were determined after silencing of IL-1R8. $\mathbf{j}-\mathbf{I}$ Effects of IL-1R8 silencing on the phosphorylation level of ERK and NFKB p65 were assessed by western blotting. Significance: ${ }^{*} P<0.05$ vs. the PBS group, ${ }^{\#} P<0.05$ vs. the CAWS group, and ${ }^{\&} P<0.05$ vs. the CAWS + IL-37b group. $N=$ 6 mice per group, and each experiment was conducted at least three times.

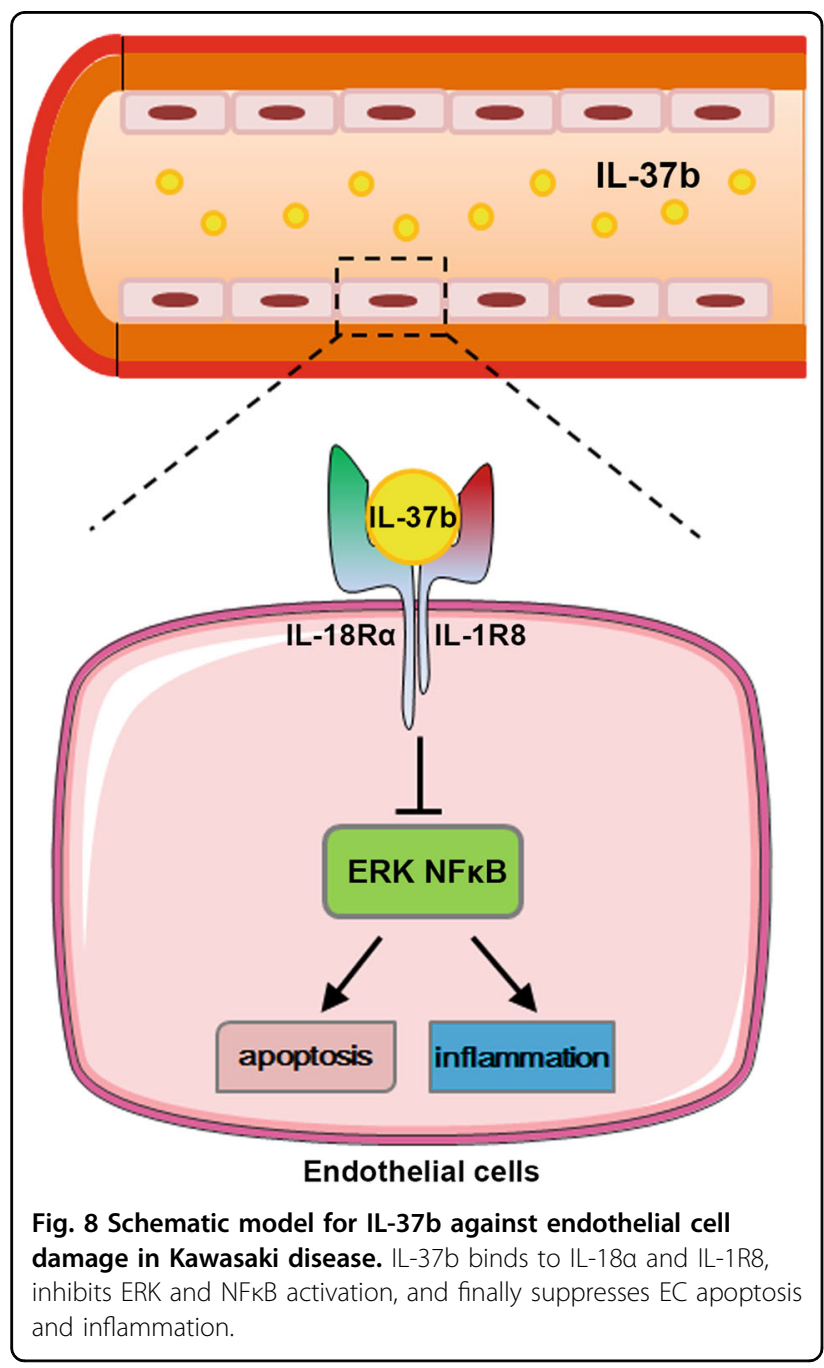

arteritis $^{33,34}$. Therefore, the protective role of IL-37b can be explained by the following assumptions. One assumption is that IL-37b improves the inflammation microenvironment in $\mathrm{KD}$, which then alleviates cytokinemediated vascular endothelial injury and subsequent inflammatory cell recruitment. Another assumption is that IL-37b directly rescues damaged vascular ECs and decreases its inflammatory response and then mitigates the immune cell recruitment and coronary inflammation in KD. Both assumptions point out that rescuing damaged ECs is essential for IL-37b to inhibit coronary arteritis in $\mathrm{KD}$.

Our following examination showed that IL-37b remarkably inhibited KD sera-induced EC apoptosis and inflammation. The anti-inflammatory activity of IL-37b has been reported in endometriosis ${ }^{35}$ and temporomandibular joint inflammation ${ }^{9}$. However, our study gave evidence for the first time that IL-37b has an anti-apoptosis effect in addition to anti-inflammatory activity. Zhang et al. reported that IL37 reduces high glucose-induced inflammation and apoptosis of podocytes via inhibiting the signal transducer and activator of transcription factor 3-Cyclophilin A (CypA) signaling pathway ${ }^{24}$. Studies have demonstrated that the recombinant IL-37 precursor binds to immobilized IL-1R8 and IL-1R8 acts as the coreceptor for IL-37, and IL-37 fails to suppress LPS-induced cytokines as well as MAPK in dendritic cells from IL-1R8-deficient mice ${ }^{25}$. To clarify the mechanism of IL-37b-suppressing apoptosis and inflammation of KD-treated ECs, the expression of IL-1R8 receptor was examined. Data showed that the expression of IL$1 \mathrm{R} 8$ was significantly upregulated in KD-treated ECs, and IL-37b supplementation further elevated its expression. Moreover, the level of surface IL-1R8 was also accordingly changed. Further study exhibited that silencing of IL-1R8 abolished the inhibition effect of IL-37b on apoptosis and inflammation, indicating that IL-37b functioned via IL-1R8 pathway.

As is known, recombinant IL-37 binds to the immobilized ligand binding $\alpha$-chain of the IL-18 receptor (IL$18 R \alpha)$ as well as to the receptor IL-1R8 to mediate antiinflammatory activity ${ }^{25}$. Studies reported that IL-18R $\alpha$ is also required for the anti-inflammatory activity of IL-37 in addition to IL-1R8 ${ }^{27}$. Therefore, we also examined the effect of IL-18R $\alpha$ on IL-37b-mediated EC apoptosis and inflammation. Data showed that the addition of recombinant human IL-37b significantly inhibited KD seruminduced IL-18R $\alpha$ upregulation. Moreover, overexpression 
Table 1 QRT-PCR primers used in this study.

\begin{tabular}{lll}
\hline Gene & Forward primer $\left(\mathbf{5}^{\prime} \rightarrow \mathbf{3}^{\prime}\right)$ & Reverse primer $\left(\mathbf{5}^{\prime} \boldsymbol{\rightarrow} \mathbf{3}^{\prime}\right)$ \\
\hline GAPDH & AAGAAGGTGGTAAGCAGG & GAAGGTGGAAGAGTGGGAGT \\
IL-1 $\beta$ & GAAATGATGGCTTATTACAGTGGCA & GTAGTGGTGGTCGGAGATTCGTAG \\
TNF-a & CTTGTTGCTCCTCTTTTGCTTA & CTTATTTCTCTCAATGACCCGTAG \\
\hline
\end{tabular}

of IL-18R $\alpha$ reversed the suppression of IL-37b-regulated EC apoptosis and inflammation (Fig. S1). Our results were inconsistent with previous reports, in which mRNA levels of IL-18R $\alpha$ were similar for LPS as well as LPS plus IL-37 in M1 macrophages ${ }^{25}$, indicating that IL-37 addition has no effect on LPS-induced IL-18R $\alpha$ expression. This discrepancy might result from different cell types and distinct stimuli. In addition, studies reported that silencing of IL-18R $\alpha$ results in a seemingly paradoxical increase in inflammation, which is attributed to the weakened association with IL-1R $8^{36}$. However, in our present study, overexpression of IL-18R $\alpha$ aggravated apoptosis and inflammation, which could be explained by the fact that IL-18 binds IL-18R $\alpha$, inducing the recruitment of IL-18R $\beta$ to form a high affinity receptor, finally triggering a proinflammatory signal into the cells terminating in $\mathrm{NF}_{\mathrm{K}} \mathrm{B}$ activation $^{37}$. These results indicate that IL-18R $\alpha$ can result in $\mathrm{EC}$ apoptosis and inflammation in $\mathrm{KD}$.

In $\mathrm{KD}$, the signaling cascades involving $\mathrm{NF}_{K} \mathrm{~B}$, and JNK and ERK MAPKs were activated ${ }^{38}$. Li et al. reported that IL-37 inhibits LPS-induced inflammation in macrophage via reducing the activation of $\mathrm{p} 38$, ERK, and $\mathrm{JNK}^{25}$. Another study demonstrated that IL-37 suppresses inflammation via inhibiting NFKB and ERK1/2 signaling pathway $^{39}$. Luo et al. revealed that IL-37b can inhibit p38, ERK, JNK, and NFKB p65 activation in response to IL- $1 \beta^{9}$. The three MAPK protein kinases and $\mathrm{NF}_{K} \mathrm{~B}$ are reported to participate in regulating apoptotic process and inflammatory response ${ }^{40-42}$. Therefore, we examined the phosphorylation level of ERK, JNK, p38, and NFKB p65. Results displayed that ERK and NFKB p65 were activated after KD sera treatment, which were suppressed after IL$37 \mathrm{~b}$ addition. NFKB p65 translocation observation further demonstrated that IL-37b could inhibit NFKB p65 activation, suggesting that IL-37b might suppress EC apoptosis and inflammation via downregulating ERK and NFkB signaling pathway.

According to the above analysis, our data provide brand new evidence that IL-37b has a markedly protective effect on KD. Our results demonstrate that IL-37b addition can inhibit EC apoptosis and inflammation via the receptor IL-1R8, which causes inactivation of ERK and NFKB, finally alleviating KD coronary arteritis. Given that KD is clinically treated mainly through IVIG plus aspirin, which is expensive and still at risk to develop CAA exploring new alternative treatment drugs or strategies would be extremely valuable to the management of $\mathrm{KD}$ patients. Our new findings suggest that IL-37b may be a good candidate for inhibiting EC damage in KD. This exciting new opportunity awaits further deep research and subsequent clinical translation. However, some limitations exist in our present study. Whether other IL-37 variants also function in KD remains uncertain and requires further investigation. Nevertheless, our current study exhibits novel evidence that IL-37b is a very effective candidate drug to treat KD.

\section{Materials and methods \\ Patients' blood sample and ethical consideration}

Collection of patients' blood samples were performed according to our previous report ${ }^{7}$. All the harvested sera samples were regularly stored at $-80^{\circ} \mathrm{C}$ within $4 \mathrm{~h}$ after collection for later use. Moreover, all the participants gave written informed consent for using their clinical information and blood samples for academic research. This research was authorized by the ethics committee of Wenzhou Medical University, and conducted in accordance with the Helsinki Declaration.

\section{Enzyme-linked immunosorbent assay}

Serum concentrations of IL-37 were determined by the corresponding ELISA kits according to the manufacturer's instructions. ELISA kit for human IL-37 was purchased from Solarbio (Beijing, China).

\section{Quantitative real-time polymerase chain reaction}

Cells and/or tissues were harvested and lysed in Trizol. Total RNA was collected and reverse-transcribed using a PrimeScript $^{\mathrm{TM}}$ RT Reagent Kit with gDNA Eraser (Takara) to obtain cDNA. Next, real-time PCR was performed on Applied Biosystems QuantStudio 3 real-time PCR system (ThermoFisher) with 40 cycles using Power TB green PCR Master Mix (Takara). Glyceraldehyde 3-phosphate dehydrogenase (GAPDH)-specific primers were used as an internal control. The expression of all the target genes was normalized to GAPDH, and the relative change of gene expression was calculated using $2^{-\Delta \Delta C T}$ method. The primers used in this study are shown in Table 1. 


\section{Western blot analysis}

The heart and HUVECs were collected and lysed using protein extraction reagents. Then the complex was centrifuged at 12,000 rpm for $20 \mathrm{~min}$ at $4{ }^{\circ} \mathrm{C}$, and the supernatant was quantified with BCA reagents. The obtained proteins were separated on a 10 or $12 \%$ gel and transferred onto a polyvinylidene difluoride membrane. After blocking with $5 \%$ skimmed milk in TBST (TBS with $0.05 \%$ tween 20) for $2 \mathrm{~h}$, membranes were incubated with the following primary antibodies at $4{ }^{\circ} \mathrm{C}$ overnight: IL-37 (Proteintech, Chicago, USA, 1:1000, Cat. No: 60296-1-lg), IL-1R8 (Proteintech, Chicago, USA, 1:1000, Cat. No: 27828-1-AP), ATP1A1 (Proteintech, Chicago, USA, 1:10,000, Cat. No: 14418-1-AP), p-ERK (Affinity Biosciences, Cincinnati, OH, USA, 1:1000, Cat. No: AF1015), p-JNK (Affinity Biosciences, Cincinnati, OH, USA, 1:1000, Cat. No: AF3318), p-p38 (Affinity Biosciences, Cincinnati, OH, USA, 1:1000, Cat. No: AF4001), p-NFkB p65 (Affinity Biosciences, Cincinnati, OH, USA, 1:1000, Cat. No: AF2006), BAX (Signalway Antibody, California, USA, 1:1000, Cat. No: 40635), Bcl-2 (Signalway Antibody, California, USA, 1:1000, Cat. No: 40639), and GAPDH (Proteintech, Chicago, USA, 1:2000, Cat. No: 60004-1-lg). After washing with TBST for three times, the membranes were treated with horseradish peroxidase (HRP)-conjugated secondary antibodies $(1: 10,000)$ for $2 \mathrm{~h}$ at room temperature. Signals were visualized by ChemiDoc XRS + Imaging System (Bio-Rad Laboratories, Hercules, CA, USA). The densitometric values of bands on western blot were obtained by the Image J software and were subjected to statistical analysis.

\section{Preparation of CAWS}

As reported before, CAWS were prepared using $C$. albicans strain NBRC1385 ${ }^{16,43}$. In brief, C. albicans were cultured in C-limiting medium at $27^{\circ} \mathrm{C}$ for 2 days at a rotation speed of $170 \mathrm{rpm}$. Next, equal volume of ethanol was added and kept in the refrigerator at $4{ }^{\circ} \mathrm{C}$ overnight. After that, the cultures were collected by centrifugation, and the pellets were dissolved in water and stirred for $2 \mathrm{~h}$ followed by centrifugation again. Next, the soluble part was obtained, mixed with equal volume of ethanol at $4{ }^{\circ} \mathrm{C}$ overnight, and centrifuged again to obtain the pellets. Finally, the pellets were collected by acetone and kept undisturbed overnight. The obtained CAWS were dissolved in phosphate-buffered saline (PBS) buffer and autoclaved before use.

\section{Animals and experimental design}

For animal models of KD, male C57BL/6 mice at the age of 3-4 weeks were purchased from Wenzhou Medical University, License No. SCXK[Z]]2005-0019. All experimental procedures for animal studies were approved by the ethics committee of Wenzhou Medical University and performed in accordance with the Guide for the Care and Use of Laboratory Animals. Animals were housed at $23 \pm$ $2{ }^{\circ} \mathrm{C}$ with humidity of $50 \pm 5 \%$. Mice were randomly divided into four groups ( $n=6$ for each group): PBS group, CAWS group, CAWS + IL-37b group, CAWS + IL-37b + AAV9-IL-1R8 siRNA group. The mice in the CAWS (4 mg/body) treated-group were injected intraperitoneally once a day for 5 days, while mice in the PBS group were injected with PBS buffer. Recombinant protein IL-37b ( $40 \mu \mathrm{g} / \mathrm{kg}$ body weight) was intraperitoneally administered into mice 2 days before and after CAWS addition. After 4 weeks of final CAWS injection, the mice were anesthetized and sacrificed for collection of heart tissues and follow-up examinations.

\section{Hematoxylin and eosin (H\&E) staining and IHC staining}

Mice hearts were obtained and then fixed with $4 \%$ paraformaldehyde and embedded with paraffin. Five- $\mu \mathrm{m}-$ thick heart sections were dewaxed and hydrated, then stained with $\mathrm{H} \& \mathrm{E}$ solution, and observed under light microscope. IHC staining of F4/80, neutrophil, and VCAM-1 was performed as follows. Sections were dewaxed and hydrated, then incubated in $3 \% \mathrm{H}_{2} \mathrm{O}_{2}$ for 15 min. After blocking with 5\% bovine serum albumin (BSA) at $37^{\circ} \mathrm{C}$ for $30 \mathrm{~min}$, primary antibody was incubated overnight at $4{ }^{\circ} \mathrm{C}$. Next, HRP-conjugated secondary antibodies were used for detection. Diaminobenzidine was used as substrate for color development. All slides were counterstained with hematoxylin. Images of histologically stained sections were obtained using light microscope.

\section{Cells culture and treatment}

In this study, HUVECs and human monocytic leukemia cell line, THP1, were utilized. These cell lines were purchased from American Type Culture Collection (ATCC, Manassas, VA, USA) and authenticated by the Genetic Testing Biotechnology Corporation (Suzhou, China) and the KeyCen BioTech (Nanjing, China) through short tandem repeat markers. There was no mycoplasma contamination. HUVECs and THP1 were routinely cultured as reported before ${ }^{16}$. In the coculture system, HUVECs were cultured in the lower chamber, and THP1 cells were placed in the upper chamber that permits diffusion of soluble molecules. Then KD or HC sera were added to the upper chamber and treated THP1 cells for $24 \mathrm{~h}$. At the same time, the HUVECs in the lower chamber would be affected by the diffusing molecules. All cultures were grown in Dulbecco's modified Eagle's medium (DMEM). If necessary, the HUVECs would be pretreated with IL$37 \mathrm{~b}$ protein (R\&D Systems, Minneapolis, MN, USA) for $1 \mathrm{~h}$ and then co-cultured with THP1 cells for $24 \mathrm{~h}$. All cultures were grown in $\mathrm{HC}$ serum or $\mathrm{KD}$ serum that was diluted in DMEM. 


\section{Immunofluorescence staining}

After dewaxing and hydration, the heart sections were incubated in $3 \% \mathrm{H}_{2} \mathrm{O}_{2}$ for 15 min and then blocked with $5 \% \mathrm{BSA}$ at $37^{\circ} \mathrm{C}$ for $30 \mathrm{~min}$. Subsequently, the sections were incubated with primary antibodies against p-ERK, TNF- $\alpha$, and CD31 overnight at $4{ }^{\circ} \mathrm{C}$ followed by incubation at $37^{\circ} \mathrm{C}$ with Alexa Fluor 647 or Alexa Fluor 488 (1:1000, Abcam) for $1 \mathrm{~h}$. For HUVECs, the cells were fixed in $4 \%$ paraformaldehyde (PFA) for $30 \mathrm{~min}$ and blocked with $5 \% \mathrm{BSA}$ for $30 \mathrm{~min}$ at $37^{\circ} \mathrm{C}$. Then the cells were incubated with the primary antibody against $\mathrm{NF \kappa B}$ p65 overnight at $4{ }^{\circ} \mathrm{C}$. The Alexa Fluor 488 secondary antibody was applied at a 1:1000 dilution for $1 \mathrm{~h}$. Nuclei were labeled with 4,6-diamidino-2-phenylindole (DAPI). The images were captured under confocal laser microscopy (Nikon, Tokyo, Japan).

\section{DNA fragmentation evaluation}

TUNEL staining was used to detect DNA fragmentation of ECs. ECs were cultured on coverslips and treated with serum. After fixation with 4\% PFA and permeabilization with $0.3 \%$ Triton $\mathrm{X}-100$, cells were washed with PBS, incubated with TUNEL reaction mixture at $37^{\circ} \mathrm{C}$ in the dark for $1 \mathrm{~h}$, and stained by DAPI. Finally, the images were scanned and captured under confocal laser microscopy (Nikon, Tokyo, Japan).

\section{Statistical analysis}

Every experiment was repeated at least three times, and data are expressed as mean $\pm \mathrm{SD}$. All the data were analyzed using SPSS version 17.0, and they were normally distributed. For statistical significance analysis, a twotailed unpaired Student's $t$ test was performed to compare two experimental groups, and one-way analysis of variance test followed by Duncan's multiple range test was conducted for the comparison of more than two groups. $P$ values $<0.05$ were regarded as statistically significant. Sample size was chosen based on previous studies ${ }^{14,15}$, which conducted similar experiments to observe significant results. Variance was similar between the groups that were being statistically compared.

\section{Acknowledgements}

We are grateful to Professor lan P. Wicks and Jacinta Hansen (Royal Melbourne Hospital \& University of Melbourne) for the methods of preparing CAWS.

\section{Author contributions}

C.J. and Y.Z. designed the experiments. C.J., Y.Z., and S.Z. contributed to most of the experiments and data analysis. C.J., C.Ni., and L.W. contributed to protein preparation and data analysis. R.W., C.Niu., Z.W., and X.R. contributed to animal experiments. C.J., H.Q., and M.C. wrote and edited the manuscript. H.Q. and M. C. supervised all the experiments. All authors agree to be accountable for the content of the work.

\section{Funding}

This research was supported by the Natural Science Foundation of China (No. 82000469, No. 81970435, No. 81770502, and No. 81900240), Zhejiang Provincial
Natural Science Foundation of China (No. LY18H020012), Zhejiang Provincial Science and Technology Project of Traditional Chinese Medicine (No. 2018ZZ019), Wenzhou Science \& Technology Bureau Foundation (No. Y20190064), and Zhejiang Provincial Medicine and Health Technology Project (No. 2021KY801).

\section{Ethics statement}

Animal experiments were performed in accordance with the Guide for the Care and Use of Laboratory Animals and were approved by the ethics committee of Wenzhou Medical University.

\section{Conflict of interest}

The authors declare no competing interests.

\section{Publisher's note}

Springer Nature remains neutral with regard to jurisdictional claims in published maps and institutional affiliations.

Supplementary information The online version contains supplementary material available at https://doi.org/10.1038/s41419-021-03852-z.

Received: 24 January 2021 Revised: 18 May 2021 Accepted: 19 May 2021 Published online: 03 June 2021

\section{References}

1. Cavalli, G. et al. Treating experimental arthritis with the innate immune inhibitor interleukin-37 reduces joint and systemic inflammation. Rheumatology 56, 2256 (2017)

2. Sakai, N. Van et al. Interleukin-37 reduces liver inflammatory injury via effects on hepatocytes and non-parenchymal cells. J. Gastroenterol. Hepatol. 27, 1609-1616 (2012).

3. Yang, Z. et al. Role of IL-37 in cardiovascular disease inflammation. Can. J. Cardiol. 35, 923-930 (2019).

4. Chai, M. et al. The protective effect of interleukin-37 on vascular calcification and atherosclerosis in apolipoprotein E-deficient mice with diabetes. J. Interferon Cytokine Res. 35, 530-539 (2015).

5. Zeng, Q. et al. Interleukin-37 suppresses the osteogenic responses of human aortic valve interstitial cells in vitro and alleviates valve lesions in mice. Proc. Natl Acad. Sci. USA 114, 1631-1636 (2017).

6. Zhu, R. et al. Interleukin-37 and dendritic cells treated with interleukin-37 plus troponin I ameliorate cardiac remodeling after myocardial infarction. J. Am. Heart Assoc. 5, e004406 (2016).

7. Wu, B. et al. Interleukin-37 ameliorates myocardial ischaemia/reperfusion injury in mice. Clin. Exp. Immunol. 176, 438-451 (2014).

8. Lunding, L. et al. IL-37 requires IL-18Ra and SIGIRR/IL-1R8 to diminish allergic airway inflammation in mice. Allergy 70, 366-373 (2015).

9. Luo, P. et al. IL-37b alleviates inflammation in the temporomandibular joint cartilage via IL-1R8 pathway. Cell Prolif. 52, e12692 (2019).

10. Zhang, L., Zhang, J. \& Gao, P. The potential of interleukin-37 as an effective therapeutic agent in asthma. Respir. Res. 18, 192 (2017).

11. LV, J. et al. IL-37 inhibits IL-4/IL-13-induced CCL11 production and lung eosinophilia in murine allergic asthma. Allergy 73, 1642-1652 (2018).

12. Li, Y. et al. IL-37d Negatively regulates NLRP3 transcription via receptormediated pathway and alleviates DSS-induced colitis. Inflamm. Bowel Dis. 27 84-93 (2021)

13. Riva, F. et al. TIR8/SIGIRR is an interleukin-1 receptor/toll like receptor family member with regulatory functions in inflammation and immunity. Front. Immunol. 3, 322 (2012).

14. Chu, M. et al. Bone marrow-derived Micro RNA-223 works as an endocrine genetic signal in vascular endothelial cells and participates in vascular injury from Kawasaki disease. J. Am. Heart Assoc. 6, e004878 (2017).

15. He, M. et al. miR-483 Targeting of CTGF suppresses endothelial-tomesenchymal transition: therapeutic implications in Kawasaki disease. Circ Res. 120, 354-365 (2017).

16. Jia, C. et al. Endothelial cell pyroptosis plays an important role in Kawasaki disease via HMGB1/RAGE/cathespin B signaling pathway and NLRP3 inflammasome activation. Cell Death Dis. 10, 778 (2019). 
17. Yan, $X$. et al. Interleukin-37: the effect of anti-inflammatory response in human coronary artery endothelial cells. Mediat. Inflamm. 2019, 2650590 (2019).

18. Li, B. et al. Interleukin-37 attenuates lipopolysaccharide (LPS)-induced neonatal acute respiratory distress syndrome in young mice via inhibition of inflammation and cell apoptosis. Med. Sci. Monit. 26, e920365 (2020).

19. Li, C., Zhao, P., Sun, X., Che, Y. \& Jiang, Y. Elevated levels of cerebrospinal fluid and plasma interleukin-37 in patients with Guillain-Barré syndrome. Mediat. Inflamm. 2013, 639712 (2013).

20. Fujita, H., Inoue, Y., Seto, K., Komitsu, N. \& Aihara, M. Interleukin-37 is elevated in subjects with atopic dermatitis. J. Dermatol. Sci. 69, 173-175 (2013).

21. Chen, Y. et al. Endothelial Nlrp3 inflammasome activation associated with lysosomal destabilization during coronary arteritis. Biochim. Biophys. Acta Mol. Cell Res. 1853, 396-408 (2015).

22. McCrindle, B. W. et al. Diagnosis, treatment, and long-term management of Kawasaki disease: a scientific statement for health professionals from the American Heart Association. Circulation 135, e927-e999 (2017).

23. Wang, $X$. et al. MiR-223-3p alleviates vascular endothelial injury by targeting IL6ST in Kawasaki disease. Front. Pediatr. 7, 288 (2019).

24. Zhang, X., Zhu, Y., Zhou, Y. \& Fei, B. Interleukin 37 (IL-37) reduces high glucoseinduced inflammation, oxidative stress, and apoptosis of podocytes by inhibiting the STAT3-Cyclophilin A (CypA) signaling pathway. Med. Sci. Monit. 26, e922979 (2020).

25. Li, S. et al. Extracellular forms of IL-37 inhibit innate inflammation in vitro and in vivo but require the IL-1 family decoy receptor IL-1R8. Proc. Natl Acad. Sci. USA 112, 2497-2502 (2015).

26. Liu, T. et al. IL-37 inhibits the maturation of dendritic cells through the IL1R8-TLR4-NF-kB pathway. Biochim. Biophys. Acta Mol. Cell Biol. 1864, 1338-1349 (2019).

27. Nold-Petry, C. A. et al. IL-37 requires the receptors IL-18Ra and IL-1R8 (SIGIRR) to carry out its multifaceted anti-inflammatory program upon innate signal transduction. Nat. Immunol. 16, 354-365 (2015).

28. Ajibade, A., Wang, H. \& Wang, R. Cell type-specific function of TAK1 in innate immune signaling. Trends Immunol. 34, 307-316 (2013).

29. McNamee, E. N. et al. Interleukin 37 expression protects mice from colitis. Proc. Natl Acad. Sci. USA 108, 16711-16716 (2011).

30. Offenbacher, S. et al. GWAS for Interleukin-1 $\beta$ levels in gingival crevicular fluid identifies IL37 variants in periodontal inflammation. Nat. Commun. 9, 3686 (2018).

31. Cavalli, G. \& Dinarello, C. A. Suppression of inflammation and acquired immunity by IL-37. Immunol. Rev. 281, 179-190 (2018).
32. Zhao, M. et al. IL-37 isoform D downregulates pro-inflammatory cytokines expression in a Smad3-dependent manner. Cell Death Dis. 9, 582 (2018).

33. Leung, D. The potential role of cytokine-mediated vascular endothelial activation in the pathogenesis of Kawasaki disease. Acta Paediatr. Jpn. 33, 739-744 (1991).

34. Shulman, S. \& Rowley, A. Kawasaki disease: insights into pathogenesis and approaches to treatment. Nat. Rev. Rheumatol. 11, 475-482 (2015).

35. He, $Y$. et al. Interleukin-37b inhibits the growth of murine endometriosis-like lesions by regulating proliferation, invasion, angiogenesis and inflammation. Mol. Hum. Reprod. 26, 240-255 (2020)

36. Nold-Petry, C. A. et al. Increased cytokine production in interleukin-18 receptor a-deficient cells is associated with dysregulation of suppressors of cytokine signaling. J. Biol. Chem. 284, 25900-25911 (2009).

37. Kaplanski, G. Interleukin-18: Biological properties and role in disease pathogenesis. Immunol. Rev. 281, 138-153 (2018).

38. Ma, L., Guan, Y. \& Du, Z. Salvianolic acid B down-regulates matrix metalloproteinase-9 activity and expression in tumor necrosis factor-ainduced human coronary artery endothelial cells. Chin. Med. J. 128, 2658-2663 (2015).

39. Meng, P. et al. IL-37 alleviates house dust mite-induced chronic allergic asthma by targeting TSLP through the NF-KB and ERK1/2 signaling pathways. Immunol. Cell Biol. 97, 403-415 (2019).

40. Ou, L. et al. The mechanisms of graphene-based materials-induced programmed cell death: a review of apoptosis, autophagy, and programmed necrosis. Int. J. Nanomed. 12, 6633-6646 (2017).

41. Subhashini, Chauhan, P. S., Dash, D., Paul, B. N. \& Singh, R. Intranasal curcumin ameliorates airway inflammation and obstruction by regulating MAPKinase activation (p38, Erk and JNK) and prostaglandin D2 release in murine model of asthma. Int. Immunopharmacol. 31, 200-206 (2016).

42. Raish, M. et al. Momordica charantia polysaccharides ameliorate oxidative stress, inflammation, and apoptosis in ethanol-induced gastritis in mucosa through NF-kB signaling pathway inhibition. Int. J. Biol. Macromol. 111, 193-199 (2018).

43. Stock, A. T., Hansen, J. A., Sleeman, M. A., McKenzie, B. S. \& Wicks, I. P. GM-CSF primes cardiac inflammation in a mouse model of Kawasaki disease. J. Exp. Med. 213, 1983-1998 (2016). 